\title{
Elementos determinantes del compromiso organizacional en un hospital universitario de tercer nivel de atención (Determinants of organizational commitment in a third level university hospital)
}

\section{Sonia Lozano-Quintanilla Adriana Segovia-Romo*}

\begin{abstract}
This paper seeks to identify the elements that manage to have influence in the organizational commitment among professional medical employee of a third level university hospital. Professional medical employee plays an important role in the hospital environment and is an essential part of the organization management results, as well as the service provided. The background shows how some elements, such as attitudes and individual perceptions, have been related with organizational commitment in different contexts. The results obtained in this research support the hypotheses raised, corresponding to the positive relationships between the attitudes and individual perceptions with the organizational commitment of the professional medical employee in a third level university hospital.
\end{abstract}

Key words: job satisfaction, organizational commitment, professional medical employee, university hospital

JEL: J28, M12.

\footnotetext{
- Universidad Autónoma de Nuevo León, Facultad de Contaduría Pública y Administración, Av. Universidad S/N, San Nicolás de los Garza, N.L., México, C.P. 66455, (+52) 81832940 00, sonia.lozanog@gmail.com

* Universidad Autónoma de Nuevo León, Facultad de Contaduría Pública y Administración, Av. Universidad S/N, San Nicolás de los Garza , N.L., México, C.P. 66455, (+52) 81832940 00, adrianasegovia@hotmail.com
} 
Resumen. Esta investigación busca identificar los elementos que logran tener influencia en el compromiso organizacional entre el personal profesional sanitario de un hospital universitario de tercer nivel de atención. El personal profesional sanitario juega un papel importante en el ámbito hospitalario y forma parte esencial de los resultados de la organización, así como del servicio que se brinda. Los antecedentes muestran cómo se han visto relacionados algunos elementos, como actitudes y percepciones individuales, con el compromiso organizacional en distintos contextos. Los resultados obtenidos en este trabajo dan soporte a las hipótesis planteadas, correspondientes a las relaciones positivas entre las actitudes y percepciones individuales con el compromiso organizacional del personal profesional sanitario en un hospital universitario de tercer nivel de atención.

Palabras clave: compromiso organizacional, hospital universitario, personal profesional sanitario, satisfacción laboral

\section{Introducción}

El clima organizacional es el resultado de la percepción que comparten los individuos en una organización en relación a su ambiente laboral (Reinoso y Araneda, 2007). Cada individuo desarrolla su percepción a partir de ciertos elementos inherentes a la organización, los cuales no se pueden medir directamente pero pueden ser gestionados por la misma organización.

Esta situación orienta, entre los individuos pertenecientes a una organización, al despertar de un comportamiento que se refleja en sus resultados individuales y en la actividad de la organización misma.

En relación a lo anterior se encuentra el compromiso organizacional, campo importante dentro del estudio del comportamiento de los individuos en las organizaciones. El concepto de compromiso organizacional se relaciona con la empatía que mantiene el empleado con la organización en la que labora y el anhelo por seguir perteneciendo a ésta (Arias, 2006). Meyer y Allen (1997) manifestaron que el concepto de compromiso es multidimensional y por ello lo clasificaron en tres tipos de compromiso: afectivo, normativo y de continuidad.

El compromiso afectivo hace referencia al grado de afinidad que un individuo mantiene con la organización por gusto propio. El compromiso normativo se relaciona con la afinidad que existe entre un individuo y su organización cuando el individuo se siente obligado a pertenecer a la organización. Por último, el compromiso de continuidad se presenta cuando 
para un individuo, el hecho de dar por terminada la relación con la organización determina una gran pérdida para sí mismo (Meyer y Allen, 1997); un ejemplo es la atadura a la organización por el bienestar económico.

Sobre la base anterior se desprende que el grado de compromiso depende, en parte, de elementos propios de la organización en la que se desenvuelve el individuo, de la sociedad a la que pertenece y de las condiciones y el pensamiento crítico individual. A partir del sentido de compromiso en un individuo, se desarrolla una conexión psicológica entre él y su entorno social, lo que le permite adaptarse a las particularidades de cada entorno (Meyer, Becker y Van Dick, 2006).

Por consiguiente, el compromiso organizacional se genera a partir del bienestar psicológico del personal. Esta relación aproxima a la idea de que el compromiso se puede ver afectado directamente por el grado de satisfacción laboral, otro elemento perteneciente al estudio del comportamiento organizacional, convirtiéndose ambos conceptos en variables interdependientes (Mañas, Salvador, González y Agulló, 2007; Mosadegh y De Moraes, 2009). Por tanto, para que exista el compromiso organizacional es necesaria también la presencia de un alto grado de satisfacción laboral (Arias, 2006). En referencia a esta relación, Kim (2004) presenta el hallazgo de una relación positiva entre las variables de satisfacción laboral y compromiso organizacional $(r=0.629, p<.01)$ en empleados gubernamentales de Corea.

Siguiendo con las relaciones entre el compromiso organizacional y otras variables del entorno laboral, en Canadá se realizó el estudio de algunos componentes del compromiso organizacional en el que se tomaron en cuenta algunas variables como el lazo emocional, la identificación y la participación dentro de la organización, entre otras (Meyer, Paunone, Gellatly, Goffin y Jackson, 1989). Otro trabajo realizado en México por Betanzos, Andrade y Paz (2006), entre 233 empleados de organizaciones públicas y privadas, mostró el compromiso organizacional como un constructo multidimensional en el que se pueden distinguir dos componentes de las percepciones laborales entre el personal: la identificación y el afecto por la organización.

Cierto es que el compromiso organizacional es un constructo de valor en cualquier organización, y se ha estudiado en distintos contextos laborales, por tal motivo, es importante considerar que el contexto de esta investigación 
corresponde a un hospital universitario de tercer nivel de atención, el cual tiene ciertas características que lo diferencian de otro tipo de hospitales. Un hospital universitario, u hospital-escuela, está comprometido y dedicado a la asistencia, docencia e investigación médica, enfocándose en la formación teórico-práctica de estudiantes de pregrado y posgrado (Martínez, 2002).

Los hospitales se clasifican de acuerdo a su nivel de atención o complejidad, ya sea como hospitales de primer nivel, segundo nivel o tercer nivel. Este último se destaca por realizar prácticas médicas y quirúrgicas con presencia de subespecialidades, trabajando con un gran número de recursos humanos y de equipamiento, así como realizando procedimientos complejos con ayuda de gran tecnología (Julio, Vacarezza, Álvarez y Sosa, 2011).

Las prácticas docentes y de investigación llevan a la exigencia de un excelente servicio de atención y por ello apuntan a una excelente calidad y a la mejora continua del servicio, todo esto a raíz de la aplicación de los avances médicos en investigación. Debido a que los hospitales universitarios de tercer nivel de atención cuentan con las características anteriormente señaladas, en ellos se presentan ciertas condiciones laborales que incurren en el compromiso del personal y en las responsabilidades inherentes al puesto laboral, viéndose reflejadas estas actitudes en el servicio que ofrece la organización (Medina, Medina y Merino, 2015).

Es relevante también mencionar las características del personal profesional sanitario que labora en un hospital universitario. Primeramente, entre el personal profesional sanitario se puede encontrar un gran número de profesionales de distintas ramas del área médica, por ejemplo enfermeros, médicos, químicos clínicos, entre otros.

Las actividades del personal profesional sanitario que labora para un hospital universitario de tercer nivel de atención requieren gran responsabilidad y desgaste físico, ya que al adquirir la responsabilidad de trabajar por largas jornadas laborales se ven comprometidos no solamente a desenvolverse con ética y profesionalismo ante los pacientes que solicitan la atención médica, sino además, tienen la responsabilidad de colaborar en la formación práctica-profesional de los universitarios en sus respectivas áreas de experticia. En otras palabras, la responsabilidad y carga de trabajo es más elevada para quienes laboran en hospitales universitarios, en especial los de tercer nivel de atención, que para quienes se limitan solamente al brindar servicios asistenciales en otro tipo de hospitales. 
Por las razones anteriores es que esta investigación tiene como objetivo la identificación de las actitudes y percepciones individuales que ayudan a determinar el compromiso organizacional del personal profesional sanitario en un hospital universitario de tercer nivel de atención.

Concluyendo con lo anterior se define la pregunta de investigación: ¿qué elementos determinan el compromiso organizacional del personal profesional sanitario en un hospital universitario de tercer nivel de atención?

Los resultados de esta investigación ofrecen una contribución teórica a lo que actualmente se conoce referente al compromiso organizacional en los hospitales universitarios de México. Por esta cuestión es imprescindible mencionar que la unidad de estudio para este trabajo es el personal profesional sanitario de uno de los hospitales universitarios más grandes y desarrollados a nivel nacional, lo que sustenta la trascendencia de esta investigación.

A pesar de que existen muchas investigaciones sobre el tema de compromiso organizacional, hasta el momento ha sido poco lo encontrado respecto a trabajos semejantes dirigidos a los hospitales, especialmente a los hospitales universitarios en México. Lo anterior apoya la relevancia de llevar a cabo la investigación pues Rositas, Alarcón y Badii (2006), al igual que Creswell (2009), mencionan que la identificación de vacío o escasez de investigaciones sobre un importante fenómeno es una situación que justifica la parte práctica de un estudio científico.

\section{Marco teórico}

Profundizando en la revisión de la literatura, a continuación se presentan las definiciones de las variables de investigación, así como los diferentes estudios de las relaciones entre estas variables en otros trabajos de investigación.

\section{Compromiso organizacional}

Comenzando el compromiso organizacional, variable de interés en esta investigación, numerosos investigadores han centrado sus estudios en el compromiso relativo al trabajo. Entre ellos, Mowday, Porter y Steers (1982) definieron una clase de compromiso, el compromiso afectivo, como la fuerza 
relativa a la identificación del individuo con la organización y su participación en ella. Toro (1998) definió el compromiso organizacional como la disposición favorable a experimentar interés hacia el trabajo y hacia la empresa, que mueve a la persona a dar apoyo, a realizar esfuerzo adicional y a cumplir con sus responsabilidades.

Meyer y Herscovitch (2001) definen el compromiso como la fuerza que une a un individuo a un curso de acción que es relevante para un objetivo en particular. Para Chiang, Núñez, Martín y Salazar (2010), el compromiso organizacional es la fuerza con la que un individuo se siente vinculado a una organización y que implica el seguimiento de un curso de acción relevante para la organización.

Estos autores reúnen ciertos puntos de vista que permiten elaborar una nueva definición de compromiso organizacional, con la finalidad de emplearla en esta investigación, apegada al contexto presente. Se precisa entonces que el compromiso organizacional es la disposición favorable de un individuo a experimentar interés hacia las responsabilidades de su puesto y hacia la organización, que además le permite identificarse y vincularse con la misma.

Respecto a las relaciones que se han documentado en anteriores investigaciones entre el compromiso organizacional, el sentido de identificación y la satisfacción laboral, se señalan dos trabajos.

En el primero, Toro (1998) estudió la relación entre algunos aspectos del clima organizacional, como las relaciones interpersonales $(r=0.56)$, el sentido de pertenencia $(r=0.40)$ y la retribución $(r=0.32)$, y sus efectos en el compromiso de los empleados, encontrando relaciones altamente significativas $(p<0.0001)$ entre dichas variables. El investigador llevó a cabo el estudio en una muestra de 2,426 empleados de 11 empresas diferentes en Colombia concluyendo que el compromiso de los empleados tenía relación con sus variables de estudio, incluyendo en sentido de pertenencia a la organización.

En el segundo, López, Osca y Peiró (2007) declararon, a partir de la revisión de otros estudios empíricos, que la satisfacción laboral y el compromiso organizacional están recíprocamente relacionados. Por lo que al existir satisfacción respecto al trabajo que realiza el personal, se desarrolla el sentido de compromiso con la organización y viceversa. 
Ahora bien, para poder relacionar empíricamente, en este contexto, el compromiso organizacional del personal profesional sanitario con las demás variables, y además medirlo, es necesario continuar con la definición y análisis teórico de los elementos organizacionales que conllevan al desarrollo de la percepción de agrado en el entorno laboral.

\section{Satisfacción laboral}

La satisfacción laboral es uno de los campos que se han relacionado con el compromiso organizacional, aunque no en gran cantidad de trabajos de investigación. La definición más popular del concepto de satisfacción laboral es la de Locke (1969), quien la definió como una respuesta emocional placentera que resulta de la percepción subjetiva de las experiencias personales en el lugar de trabajo. Bravo, Peiró y Rodríguez (1996) definieron la satisfacción laboral como una actitud o conjunto de actitudes desarrolladas por la persona hacia su situación de trabajo, actitudes que pueden ir orientadas hacia el trabajo en general o hacia aspectos específicos del mismo.

De nuevo, los autores presentan definiciones diferentes aunque con puntos de vista muy similares. Para fines de esta investigación se utilizó una adaptación de la definición de satisfacción laboral de Bravo et al. (1996): una actitud o conjunto de actitudes desarrolladas por un individuo hacia su situación de trabajo y que pueden ir orientadas hacia el trabajo o hacia aspectos específicos del mismo.

Algunos de los trabajos que han presentado relaciones entre la satisfacción laboral y el compromiso organizacional muestran que ambas variables están interrelacionadas, pues estando presente la satisfacción laboral se genera el compromiso con la organización, y viceversa (Mowday, Steers y Porter, 1979). En otro trabajo, Thumin y Thumin (2011) destacaron que la satisfacción laboral es parte esencial del clima organizacional, por lo que en relación a lo que se mencionó en la primera parte de este trabajo, el ambiente del trabajo afecta el grado de satisfacción laboral generando el grado de compromiso organizacional en un individuo. 
Identificación y afecto por la organización

Otro de los elementos que se pueden relacionar con el compromiso organizacional es el sentido de identificación y el afecto por la organización. Esta variable compuesta se refiere a las percepciones que se desarrollan en cada individuo en una organización. Respecto al sentido de identificación, lo que se considera es el grado de aceptación de los valores organizacionales y la identificación que una persona tiene con su puesto y su organización (Betanzos Andrade y Paz, 2006).

En cuanto al sentido de afecto por la organización, éste hace referencia a los sentimientos de agrado y felicidad que una persona tiene por pertenecer a su organización (Betanzos Andrade y Paz, 2006). Por otro lado, Shragay y Tziner (2011) mencionaron que la identificación con el trabajo es el grado de involucramiento personal del empleado en su trabajo, en un nivel psicológico.

Las definiciones presentadas en ambos trabajos proporcionan las bases para elaborar la definición de la variable identificación y afecto por la organización, conjuntando ambos sentidos en una sola variable compuesta. Se establece entonces que la identificación y afecto por la organización se refiere al grado de aceptación de los valores organizacionales y la identificación que una persona tiene con su puesto y su organización, así como los sentimientos y percepciones de agrado y felicidad que un individuo manifiesta al pertenecer a una organización.

En cuanto a los antecedentes del estudio de la identificación y afecto por la organización relacionada con la variable compromiso organizacional, los trabajos realizados por Betanzos et al. (2006), Kim (2004) y Mowday et al. (1979) presentan la identificación con la organización y el afecto por la organización como determinantes del compromiso organizacional con base en sus resultados con relaciones positivas.

En otro trabajo, un grupo de 354 trabajadores españoles, pertenecientes a una empresa especializada como centro de contacto, participaron para evidenciar la relación entre el sentido de identificación con la organización, el afecto por la organización y el compromiso. Se incluyó personal de puestos de mando, agentes teleoperadores y sus superiores inmediatos. Resultaron correlaciones significativas entre el sentido de 
identificación $(r=-0.245, p<0.01)$ y afecto por la organización $(r=-0.283, p$ < 0.01) con el compromiso organizacional (Dávila y Jiménez, 2014).

Las investigaciones señaladas en los párrafos anteriores permiten elaborar el modelo gráfico que muestra las relaciones propuestas entre las variables de estudio de esta investigación (Figura 1).

Figura 1. Modelo gráfico propuesto.

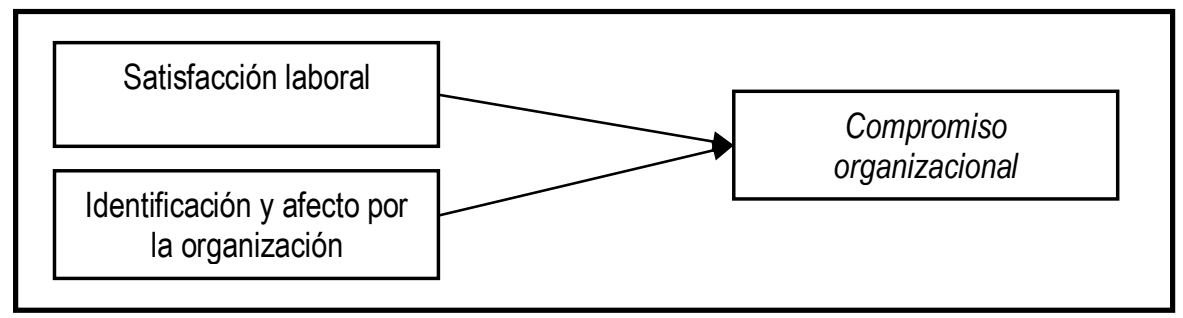

Fuente: Elaboración propia de los autores.

Por consiguiente, con base en el modelo gráfico propuesto, apoyado por la literatura revisada, se desarrollaron dos hipótesis de investigación:

$\mathrm{H}_{1}$ : La satisfacción laboral impacta positivamente en el compromiso organizacional del personal profesional sanitario.

$\mathrm{H}_{2}$ : La identificación y el afecto por la organización impactan positivamente en el compromiso organizacional del personal profesional sanitario.

\section{Método}

Esta investigación tiene un enfoque cuantitativo, es de tipo explicativo, no experimental y transversal. Se tomó como unidad de análisis al personal profesional sanitario de un hospital universitario de tercer nivel de atención del noreste de México.

Como universo de estudio se consideró a todos los empleados del hospital universitario ya mencionado, siendo un total de 3,000 elementos. La población incluye a los siguientes profesionales sanitarios: Licenciado en Enfermería (286), Médico Cirujano Partero (122), Químico Clínico Biólogo (106), Licenciado en Nutrición (22) y Licenciado en Psicología (19) que laboraban en el hospital universitario durante el periodo del estudio de campo, siendo un total de 555 elementos. 
El criterio que se utilizó para estimar la muestra fue tomando en consideración tanto el tipo de variables, como el tipo de preguntas en el instrumento de medición y la población de estudio (Rositas, 2014). En esta investigación las variables son cuantitativas, las preguntas del instrumento de medición son de tipo Likert y la población es finita; se obtuvo como resultado una " $n$ " de 161 elementos. El muestreo realizado fue no probabilístico cuidando la proporción del número total de elementos en la población. La Tabla 1 muestra la cantidad de profesionales sanitarios requeridos para la muestra.

Tabla 5. Selección de la muestra no probabilística en proporción.

\begin{tabular}{lcc}
\hline \multicolumn{1}{c}{ Licenciatura } & $\begin{array}{c}\text { Número total de elementos } \\
\text { en la población }\end{array}$ & $\begin{array}{c}\text { Número de elementos } \\
\text { requeridos para la muestra }\end{array}$ \\
\hline Licenciado en Enfermería & $286(51.53 \%)$ & $83(51.53 \%)$ \\
$\begin{array}{l}\text { Químico Clínico Biólogo / } \\
\text { Químico Biólogo Parasitólogo / }\end{array}$ & $106(19.10 \%)$ & $31(19.10 \%)$ \\
Químico Farmacéutico Biólogo & $122(21.98 \%)$ & $35(21.98 \%)$ \\
Médico Cirujano Partero & $19(3.42 \%)$ & $6(3.42 \%)$ \\
Licenciado en Psicología & $22(3.96 \%)$ & $6(3.96 \%)$ \\
Licenciado en Nutrición & $555(100 \%)$ & $161(100 \%)$ \\
Total & &
\end{tabular}

Fuente: Elaboración propia de los autores.

\section{Elaboración del instrumento de medición}

El instrumento elaborado para la recolección de los datos se integró en dos secciones. La sección 1 recabó la información general del participante: edad, años laborando en la empresa, género, estado civil, tipo de empleado, la especificación de su licenciatura y el máximo nivel de estudios alcanzado. La sección 2 incluyó 11 reactivos, cuatro para cada una de las variables independientes y 3 para la variable dependiente.

Las respuestas para cada uno de los reactivos mantuvieron una escala Likert de 5 puntos, en donde 1 indicaba estar "Totalmente en desacuerdo", 2 "En desacuerdo", 3 "Ni en desacuerdo ni de acuerdo", 4 "De acuerdo" y 5 "Totalmente de acuerdo". 
La construcción del instrumento de medición se realizó después de una revisión de la literatura con la finalidad de encontrar escalas previamente implementadas en contextos similares o distintos pero tomando como base la confiabilidad reportada para cada uno de ellos. Luego de la elaboración del instrumento se solicitó apoyo de seis expertos en el área de capital humano para evaluar que el contenido y la redacción de los reactivos correspondieran con las definiciones de esta investigación.

\section{Análisis de confiabilidad}

El análisis de confiabilidad se ejecutó a partir de una prueba piloto a 51 profesionales sanitarios del hospital universitario en estudio para probar el instrumento de medición previamente validado. Se obtuvieron los índices de confiabilidad alfa de Cronbach para cada grupo de ítems de las variables de investigación y el índice alfa de Cronbach global del instrumento con dos softwares estadísticos: SPSS (Statistical Package for the Social Sciences) versión 21 y SmartPLS 3.2.6 (Tabla 2). Los índices alfa de Cronbach para cada variable se encontraron en un rango de 0.78 a 0.823 .

Tabla 2. Resultados del análisis de confiabilidad de las variables (Alfa de Cronbach).

\begin{tabular}{lccc}
\hline \multicolumn{1}{c}{ Variable } & $\begin{array}{c}\text { Alfa de Cronbach } \\
\text { (SPS) }\end{array}$ & $\begin{array}{c}\text { Alfa de Cronbach } \\
\text { estandarizado (SPS) }\end{array}$ & $\begin{array}{c}\text { Alfa de Cronbach } \\
\text { (SmartPLS) }\end{array}$ \\
\hline Compromiso organizacional & 0.780 & 0.781 & 0.781 \\
Identificación y afecto por la & 0.791 & 0.792 & 0.792 \\
organización & 0.820 & 0.823 & 0.823 \\
Satisfacción laboral & &
\end{tabular}

Fuente: Elaboración propia de los autores y formato adaptado de Segovia (2014).

El índice alfa de Cronbach para el instrumento resultó de 0.864 , por lo que se decidió continuar con la aplicación de la encuesta para lograr la muestra estimada.

Recolección de los datos

El proceso de recolección de los datos se llevó a cabo durante seis meses, de septiembre de 2016 a febrero de 2017. Se efectuó dentro del horario 
laboral de los participantes y se trabajó en los tres turnos del hospital universitario: matutino, vespertino y nocturno. La solicitud de participación se llevó a cabo de manera personal y directa con cada uno de los elementos de la población de estudio y su participación fue totalmente voluntaria. El número total de encuestas aplicadas fue de 212 pero solamente 191 se contestaron satisfactoriamente, por lo que éstas forman el total de la muestra.

\section{Resultados}

Para el análisis de los datos recolectados se utilizó el software estadístico SmartPLS 3.2.6. En seguida se presentan los resultados mediante dos componentes: el componente del modelo de medición y el componente del modelo estructural.

\section{Componentes del modelo de medición}

Como parte de este componente se encuentran los indicadores de los modelos reflexivos, tal es el caso del modelo de este estudio, entre los que se reportan el promedio de la varianza extraída (AVE) y la confiabilidad compuesta; ésta última evalúa la consistencia interna del modelo. Hair, Ringle y Sarstedt (2011) reportan que para los modelos de medición reflexivos, los valores de AVE y de la confiabilidad compuesta deben ser mayores a 0.50 y 0.70 , respectivamente. La Tabla 3 presenta los valores obtenidos para ambos indicadores respecto a las tres variables.

Tabla 3. Resultados del análisis de medición del modelo de ítems.

\begin{tabular}{lcc}
\hline \multicolumn{1}{c}{ Variable } & AVE & Confiabilidad compuesta \\
\hline Compromiso organizacional & 0.696 & 0.873 \\
Identificación y afecto por la organización & 0.615 & 0.865 \\
Satisfacción laboral & 0.655 & 0.883 \\
\hline
\end{tabular}

Fuente: Elaboración propia de los autores utilizando el software SmartPLS 3.2.6.

Las evaluaciones de la validez de los modelos de medición reflexivos se realizan mediante la validez convergente, la cual se prueba con el AVE. Para la validez discriminante se debe cumplir con dos criterios: el criterio de Fornell-Larcker y el de las cargas cruzadas. Estos criterios permiten reflejar la capacidad de cada variable para explicar que los indicadores de cada 
variable más correlación con la misma variable que con otras variables del modelo (Hair et al., 2011).

El criterio de Fornell-Larcker postula que una variable comparte más varianza con sus indicadores que con los de otra variable del modelo. En la Tabla 4 se presentan las correlaciones entre las variables latentes y se observa que se cumple este criterio ya que las correlaciones más altas se presentan con respecto a la misma variable evaluada.

Tabla 4. Correlación entre variables latentes.

\begin{tabular}{lccc}
\hline \multicolumn{1}{c}{ Variable } & CO & Id y A & SL \\
\hline Compromiso organizacional & 0.834 & & \\
Identificación y afecto por la organización & 0.742 & 0.784 & \\
Satisfacción laboral & 0.737 & 0.622 & $\mathbf{0 . 8 0 9}$ \\
\hline
\end{tabular}

Nota: CO: Compromiso organizacional; Id y A: Identificación y afecto por la organización; SL:

Satisfacción laboral.

Fuente: Elaboración propia de los autores utilizando el software SmartPLS 3.2.6.

El criterio de las cargas cruzadas establece que la carga de cada indicador debe ser mayor respecto a la variable que evalúa que con otras variables del modelo (Hair et al., 2011). La Tabla 5 muestra las cargas obtenidas para cada uno de los ítems y se observa que se cumple también con este segundo criterio.

Tabla 5. Cargas cruzadas.

\begin{tabular}{lccc}
\hline İtem & CO & Id y A & SL \\
\hline 10CO & $\mathbf{0 . 8 5 7}$ & 0.640 & 0.687 \\
14IDAF & 0.492 & $\mathbf{0 . 7 5 2}$ & 0.474 \\
16SL & 0.672 & 0.542 & $\mathbf{0 . 8 8 2}$ \\
17CO & $\mathbf{0 . 8 6 9}$ & 0.642 & 0.638 \\
21IDAF & 0.559 & $\mathbf{0 . 7 5 3}$ & 0.515 \\
23SL & 0.572 & 0.524 & $\mathbf{0 . 7 9 5}$ \\
2SL & 0.519 & 0.443 & $\mathbf{0 . 7 7 8}$ \\
31CO & $\mathbf{0 . 7 7 5}$ & 0.574 & 0.504 \\
35IDAF & 0.654 & $\mathbf{0 . 8 4 2}$ & 0.491 \\
7IDAF & 0.606 & $\mathbf{0 . 7 8 7}$ & 0.478 \\
9SL & 0.606 & 0.498 & $\mathbf{0 . 7 7 7}$ \\
\hline
\end{tabular}

Fuente: Elaboración propia de los autores utilizando el software SmartPLS 3.2.6. 


\section{Componente del modelo estructural}

En la Figura 2 se presentan las betas de las relaciones entre las variables del modelo y la $R^{2}$ (0.674), que representa la explicación de la variable dependiente. La interpretación del valor de este indicador resulta como una explicación moderada del modelo, resultado aceptable, pues está arriba de 0.50 como lo indican Hair et al. (2011).

Figura 2. Modelo de ecuaciones - Algoritmo (SmartPLS 3.2.6).

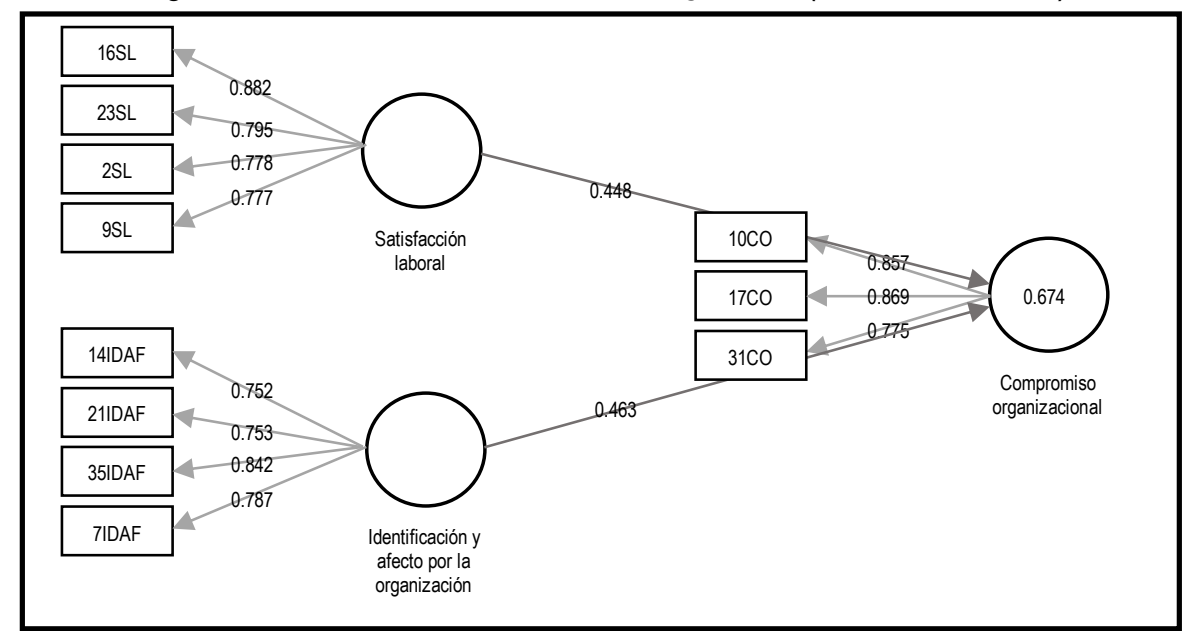

Fuente: Elaboración propia de los autores utilizando el software SmartPLS 3.2.6.

El componente del modelo estructural muestra también la significancia de las trayectorias del modelo por medio de la t de Student y el valor $p$. La Tabla 6 presenta las trayectorias y su significancia, observándose todos los resultados significativos $(t>2.0, p<0.001)$.

Tabla 6. Resultados del análisis estructural.

\begin{tabular}{lccc}
\hline \multicolumn{1}{c}{ Variable } & $\boldsymbol{b}$ & $\begin{array}{c}\boldsymbol{t} \text { de } \\
\text { Student }\end{array}$ & Valor $\boldsymbol{p}$ \\
\hline Identificación y afecto por la organización - & 0.463 & 7.427 & 0.000 \\
Compromiso organizacional & 0.448 & 6.179 & 0.000 \\
\hline
\end{tabular}

Fuente: Elaboración propia de los autores utilizando el software SmartPLS 3.2.6. 
Los resultados de esta investigación permiten comprobar las hipótesis planteadas con base en el modelo gráfico propuesto:

$\mathrm{H}_{1}$, existe relación positiva entre las variables satisfacción laboral y compromiso organizacional $(b=0.448, p<0.001)$

$\mathrm{H}_{2}$, existe relación positiva entre las variables identificación y afecto por la organización y compromiso organizacional $(b=0.463, p<0.001)$

En cuanto a la relevancia predictiva del modelo (Q2), Hair et al. (2011) señalan que se deben presentar valores mayores a cero, esto confirma la relevancia predictiva del modelo de esta investigación pues se obtuvo un valor $\mathrm{Q}^{2}$ de 0.439 .

\section{Discusión}

De acuerdo a los resultados obtenidos se muestra el cumplimiento del objetivo de la investigación y se logra contestar la pregunta de investigación. Como respuesta al objetivo, los resultados determinan que la satisfacción laboral, que es una actitud del personal, y la identificación y el afecto por la organización, que son percepciones individuales, son elementos que determinan el compromiso organizacional del personal profesional sanitario en un hospital universitario de tercer nivel de atención. De igual manera, la respuesta a la pregunta respecto a cuáles son los elementos que determinan el compromiso organizacional del personal profesional sanitario en un hospital universitario de tercer nivel de atención, indica que la satisfacción laboral y la identificación y el afecto por la organización son dichos elementos.

En cuanto a la comprobación de las hipótesis, los resultados reflejan que la satisfacción laboral y la identificación y el afecto por la organización se relacionan positivamente con el compromiso organizacional. La comprobación de la hipótesis $1\left(\mathrm{H}_{1}\right)$, impacto positivo de la satisfacción laboral en el compromiso organizacional, confirma los resultados de Kim (2004) en una investigación sobre empleados gubernamentales de Corea y las revisiones de trabajos empíricos de López et al. (2007) citados en el planteamiento del problema. Las declaraciones de Mowday et al. (1979), Mañas et al. (2007) y Mosadegh y De Moraes (2009) hablan sobre una 
interdependencia entre la satisfacción laboral y el compromiso organizacional. Esta interdependencia involucra una relación en ambas direcciones entre las dos variables, una de ellas el impacto positivo de la satisfacción laboral sobre el compromiso organizacional, lo cual respalda la relación que resultó entre esas dos variables en el contexto de este estudio.

Por otra parte, la comprobación de la hipótesis $2\left(\mathrm{H}_{2}\right)$, impacto positivo de la identificación y afecto por la organización en el compromiso organizacional, fortalece los resultados de Betanzos et al. (2006) y Dávila y Jiménez (2014), Mowday et al. (1979) y Kim (2004) los cuales muestran que el sentido de identificación y afecto por la organización es un determinante del compromiso organizacional. Betanzos et al. (2006) efectuó su estudio en México pero en un contexto diferente pues los sujetos de su estudio fueron personal administrativo y de finanzas de un aeropuerto.

Es relevante mencionar que la comprobación del modelo de esta investigación no se había realizado en otras investigaciones, lo cual cubre dos de las deficiencias teóricas que señala Creswell (2009): que los temas no hayan sido explorados en una población en particular, en este caso el contexto hospitalario en México, y que la literatura puede necesitar ser replicada o repetida para ver si los resultados se mantienen en un nuevo contexto, en referencia a los estudios previos que declaran relaciones positivas de manera independiente entre la satisfacción laboral y el compromiso organizacional y entre la identificación y afecto por la organización y el compromiso organizacional.

Este modelo demuestra que, en el ambiente de un hospital universitario, el personal profesional sanitario realmente logra verse comprometido con la organización a partir de un grado alto de satisfacción laboral y de la presencia de un sentido de identificación y afecto por la misma organización. Lo anterior sostiene la importancia de buscar que el personal del contexto hospitalario se mantenga satisfecho con los aspectos inherentes a su trabajo y sus actividades en la organización, así mismo, que el personal se sienta identificado con los valores de la organización y desarrollen una percepción de agrado y felicidad por pertenecer a la misma; esto con la finalidad de hacer crecer el sentido de compromiso que tiene el personal con la organización.

De alguna manera el compromiso organizacional se refleja en los resultados que el personal obtiene dentro de la organización, es decir, el 
compromiso organizacional se refleja en el servicio que brinda el hospital universitario (Medina et al., 2015). De lo anterior se observa la trascendencia de los resultados de esta investigación, los cuales ofrecen aportaciones a la teoría y a la práctica del tema.

Con respecto a la aportación teórica de esta investigación está la integración de la variable identificación y afecto por la organización, que engloba el grado de aceptación de los valores de la organización y el agrado al pertenecer a ella, dentro del marco teórico del compromiso organizacional. Por otro lado, la aportación práctica se refiere a que el estudio puede ayudar en la dirección del personal profesional sanitario en los ambientes que requieren de grandes responsabilidades laborales, como es el caso de un hospital universitario de tercer nivel de atención.

\section{Referencias}

Arias F. (2006). Liderazgo para la competitividad y el compromiso. En F. Arias ( $6^{\mathrm{a}}$ ed), Administración de recursos humanos: Para el alto desempeño (pp. 203-221). México: Trillas.

Betanzos, N., Andrade, P. \& Paz, F. (2006). Compromiso organizacional en una muestra de trabajadores mexicanos, Revista de Psicología del Trabajo y de las Organizaciones, 22(1), 25-33.

Bravo, M., Peiró, J. \& Rodríguez, I. (1996). Satisfacción laboral. En J. Peiró y F. Prieto (eds), Tratado de psicología del trabajo, 1. La actividad laboral en su context, (pp. 343394). España: Ed. Síntesis S.A.

Chiang, M., Núñez, A., Martín, M. \& Salazar, M. (2010). Compromiso del trabajador hacia su organización y la relación con el clima organizacional: Un análisis de género y edad, Panorama Socioeconómico, 40, 92-103.

Creswell, J. (2009). The Introduction. En J. Creswell (3a ed), Research design: Qualitative, quantitative, and mixed methods approaches. Thousand Oaks: Sage Publications.

Dávila, C. \& Jiménez, G. (2014). Sentido de pertenencia y compromiso organizacional: predicción del bienestar, Revista de Psicología, 32(2), 272-302.

Hair J., Ringle, C. \& Sarstedt, M. (2011). PLS-SEM: Indeed a Silver Bullet, Journal of Marketing Theory and Practice, 19(2), 139-152.

Julio, V., Vacarezza, M., Álvarez, C. \& Sosa, A., (2011). Niveles de atención, de prevención y atención primaria de la salud, Arch Med Interna, 33(1), 11-14.

$\mathrm{Kim}$, S. (2004). Individual-level factors and organizational performance in government organizations, Journal of Public Administration Research and Theory, 15(2), 245261. 
Locke, E. (1969). What is job satisfaction?, Organizational Behavior and Human Performance, 4, 309-336.

López, B. Osca, A. \& Peiró, J. (2007). El papel modulador de la implicación con el trabajo en la relación entre el estrés y la satisfacción laboral, Psicothema, 19(1), 81-87.

Mañas, M., Salvador, C., González, E. \& Agulló, E. (2007). La satisfacción y el bienestar psicológico como antecedentes del compromiso organizacional, Psicothema, 19(3), $395-400$.

Martínez, F. (2002). El hospital universitario, su naturaleza y sus fines, Medicina Universitaria, 4(16), 187-189.

Medina, M., Medina, M. \& Merino, L. (2015). La investigación científica como misión académica de los hospitales públicos universitarios, Revista Cubana de Salud Pública, 41(1), 139-146.

Meyer, J. \& Allen, N. (1997). Commitment in the workplace: Theory, research, and application. Newbury Park, CA: Sage.

Meyer, J., Becker, T. \& Van Dick, R. (2006). Social identities and commitments at work: toward an integrative model, Journal of Organizational Behavior, 27, 665-683.

Meyer, J. \& Herscovitch, L. (2001). Commitment in the workplace: Toward a general model, Human Resource Management Review, 11, 299-326.

Meyer, J., Paunonen, S., Gellatly, I., Goffin, R. \& Jackson, D. (1989). Organizational commitment and job performance: It's the nature of the commitment that counts, Journal of Applied Psychology, 74(1), 152-156.

Mosadegh, A. \& De Moraes, A. (2009). Factor affecting employees' job satisfaction in public hospitals: Implications for recruitment and retention, Journal of General Management, 34(4), 51-66.

Mowday, R., Porter, L. \& Steers, R. (1982). Organizational linkages: The psychology of commitment, absenteeism, and turnover. San Diego, CA: Academic Press.

Mowday, R., Steers, R. \& Porter, L. (1979). The measurement of organizational commitment, Journal of Vocational Behavior, 14, 224-247.

Reinoso, H. \& Araneda, B. (2007). Diseño y validación de un modelo de medición del clima organizacional basado en percepciones y expectativas, Revista de Ingeniería Industrial, 6(1), 39-54.

Rositas, J. (2014). Los tamaños de las muestras en encuestas de las ciencias sociales y su repercusión en la generación del conocimiento, Innovaciones de Negocios, 11(22), 235-268.

Rositas, J., Alarcón, G. \& Badii, M. (2006). El desarrollo y evaluación de la declaración del problema de investigación, Innovaciones de Negocios, 3(2), 331-345.

Segovia, A. (2014). El liderazgo, la compensación variable, el empowerment psicológico y su impacto en la efectividad del empleado: Un enfoque de modelación mediante ecuaciones estructurales (Disertación doctoral). Recuperada de la Colección Digital UANL.

Shragay, D. \& Tziner, A., (2011). The generational effect on the relationship between job involvement, work satisfaction, and organizational citizenship behavior, Revista de Psicología del Trabajo y de las Organizaciones, 27(2), 143-157. 
Thumin, F. \& Thumin, L. (2011). The measurement and interpretation of organizational climate, Journal of Psychology, 145(2), 93-109.

Toro, F. (1998). Predicción del compromiso del personal a partir del análisis del clima organizacional, Revista de Psicología del Trabajo y de las Organizaciones, 14(3), 333-344. 